\title{
Development of Au-Nanoprobes Combined with Loop-Mediated Isothermal Amplification for Detection of Isoniazid Resistance in Mycobacterium tuberculosis
}

\author{
Jutturong Ckumdee, ${ }^{1}$ Thongchai Kaewphinit, ${ }^{2}$ \\ Kosum Chansiri, ${ }^{3}$ and Somchai Santiwatanakul ${ }^{4}$ \\ ${ }^{1}$ Department of Clinical Pathology, Faculty of Medicine, Vajira Hospital, Navamindradhiraj University, Samsen Rd, \\ Bangkok 10300, Thailand \\ ${ }^{2}$ Innovative Learning Center, Srinakharinwirot University, Sukhumvit 23, Bangkok 10110, Thailand \\ ${ }^{3}$ Department of Biochemistry, Faculty of Medicine, Srinakharinwirot University, Sukhumvit 23, Bangkok 10110, Thailand \\ ${ }^{4}$ Department of Pathology, Faculty of Medicine, Srinakharinwirot University, Sukhumvit 23, Bangkok 10110, Thailand
}

Correspondence should be addressed to Thongchai Kaewphinit; thongchaika@swu.ac.th and Somchai Santiwatanakul; titi41@yahoo.com

Received 8 March 2016; Revised 25 August 2016; Accepted 28 August 2016

Academic Editor: Mostafa Khajeh

Copyright ( 2016 Jutturong Ckumdee et al. This is an open access article distributed under the Creative Commons Attribution License, which permits unrestricted use, distribution, and reproduction in any medium, provided the original work is properly cited.

\begin{abstract}
Multidrug resistant tuberculosis (MDR-TB) is Mycobacterium tuberculosis that does not respond to isoniazid and rifampicin, so the condition worsens continuously and creates difficulties for treatment by public health control programmes, especially in developing countries. The real time polymerase chain reaction (PCR) combined with agarose gel electrophoresis or strip tests is useful molecular tools for diagnosis of MDR-TB. Novel loop-mediated isothermal amplification (LAMP) can also detect drug resistance, which is a one-point mutation, by designing inner primers of $5^{\prime}$ end specific with the mutant. Au-nanoprobes on hybridisation with LAMP products containing target-specific sequences remain red, whereas test samples without specific sequences in the probe turn purple due to salt-induced aggregation of the Au-nanoprobes. In this study, a strategy was designed based on the LAMP of a DNA sample coupled to specific Au-nanoprobes, which showed the potential to provide a rapid and sensitive method for detecting isoniazid resistance at katG gene position $315(\mathrm{G} \rightarrow \mathrm{C}) .46$ clinical samples were tested and showed $100 \%$ specificity and sensitivity compared with Genotype ${ }^{\circledR}$ MDR-TB Plus. This method was advantageous because it is rapid, cheap, specific, and sensitive. Further, it does not require thermal cycles for MDR-TB detection.
\end{abstract}

\section{Introduction}

Mycobacterium tuberculosis (MTB) infection has become an emerging global health concern. According to the World Health Organisation (WHO) Global Tuberculosis Report 2012, there were an estimated 8.7 million cases of TB in 2011 [1]. Worldwide, $3.7 \%$ of newly diagnosed incidences and $20 \%$ of cases which have previously been treated are thought to be a form of tuberculosis which has developed resistance to multiple drugs (MDR-TB), defined as resistance to at least isoniazid (INH) and rifampicin (RMP). In 2011, there were an estimated 630,000 cases of MDR-TB among 12 million prevalent cases of TB [1]. The biggest challenge in any control and treatment system for TB is MDR-TB. It is estimated that MDR-TB constitutes about $5 \%$ of all TB cases worldwide. A significant parameter utilised to describe the phenotype of drug resistant strains of MTB, including MDR strains, is the enzymatic activity of the catalase-peroxidase system.

The last decade has witnessed the development of the LAMP DNA amplification approach, which can be applied instead of conventional PCR [2] and which offers the advantage of greater speed as well as being more sensitive, specific, and cost-effective $[3,4]$. Once under way, the reaction is able to proceed on the basis of the strand displacement capability of the Bst DNA polymerase. The temperature remains constant throughout, and only one enzyme is required for the 
test. Novel loop-mediated isothermal amplification (LAMP) can also detect drug resistance, which is a one-point mutation by designing an inner primer of $5^{\prime}$ end specific mutant $[5,6]$.

Nanoparticle-based assays represent a current trend in DNA detection [7]. In particular, Au-nanoparticles (AuNPs) have unique optical properties that make them suitable for the design of labelled Au-nanoprobes. They offer advantages in that they are inexpensive and afford visual detection, unlike more conventional methods of detection such as those used in fluorescence or radioactivity-based assays. Previous work in colorimetric detection approaches using Au-nanoprobes coupled to LAMP has been described [8]. Recently, the combination of LAMP amplification and AuNP probes detection has been developed successfully for detection of the mutations associated with rifampicin resistance in MTB [9]. However, this experiment conducted an analysis in terms of the absorbance ratio and assay on the spectrophotometry, so it would not be convenient for detection of INHr-TB in field samples. The instant study demonstrated that LAMP amplification combined with the Au-nanoprobes system took less than $75 \mathrm{~min}$ to yield a colorimetric result, observed for detection of INHr-TB using a suitable eye detector.

In this study, the researchers designed a strategy based on an Au-nanoprobe detection protocol combined with loopmediated isothermal amplification (LAMP) for the rapid and discriminatory detection of MDR-TB and TB strains as well as for the simultaneous characterisation of the presence of mutations associated with isoniazid resistance. In conducting field analysis, the speed and heightened specificity of LAMP, along with its isothermal profile, make this approach ideal for DNA sample amplification in comparison with standard PCR. This method is advantageous because it is swift, inexpensive, specific, and sensitive to MDR-TB detection. It could be convenient for detection of MDR-TB in field samples as well.

\section{Materials and Methods}

2.1. Sample Preparation. The 46 clinical samples were provided by the Bureau of Tuberculosis, which operates under the Department of Disease Control, Thai Ministry of Public Health. All clinical samples were identified as kat $\mathrm{G}$ gene mutations associated with INH resistance by using Genotype MDR-TB Plus (Hain Lifescience GmbH, Nehren, Germany). There were 31 samples of TB with INH resistance, 15 samples with INH susceptibility, and H37RV strain used as negative control. However, Genotype MDR-TB Plus detection was confirmed after conventional methods detected cultures on Lowenstein-Jensen (L.-J.) medium after 8 weeks. After that, all samples were extracted DNA for LAMP amplification using a modified method from Rienthong et al. [10]. Briefly, one loopful of cells was first suspended in distilled water $(300 \mu \mathrm{L})$ before undergoing $20 \mathrm{~min}$ of subsequent heat treatment at a temperature of $95^{\circ} \mathrm{C}$ in Thermoblock. Sonification was then performed for $15 \mathrm{~min}$ using an ultrasonic bath at the highest speed setting. The samples were then sent to a standard centrifuge containing an aerosol-tight rotor for 5 min spinning at $10,000 \times \mathrm{g}$. The LAMP amplification stage involved the use of a supernatant.
2.2. Primers and Probe Designed Specific katG Gene of INHr$T B$. The LAMP primers for amplification of specific kat $\mathrm{G}$ genes in MTB were synthesised from Bio Basic Canada Inc. using the manual designed and primer-designing software Primer Explorer Ver. 3 (Eiken Chemical Co., Ltd.). The primer sets consisted of outer primers (F3 and B3) and inner primers (FIP and BIP). The directions and details for the primers are shown in Table 1 and Figure 1.

All oligonucleotide DNA probes and complementary DNA targets for the detection of MDR-TB and MTB were designed based on the nucleotide sequence of kat $\mathrm{G}$ gene retrieved from NCBI (Accession number X68081.1). The gene had 4,810 base pairs. Nucleotide sequences at positions 2,9242,938 (15 bp) were selected for synthesis of the DNA probe and specificity to the DNA target of MDR-TB and MTB. For DNA probe synthesis, the linker of $d\left(A_{10}\right)$ was added to the $5^{\prime}$ end with the thiol (Table 1). The accuracy of the probe was confirmed using a BLAST nucleotide-free program provided by NCBI. The probe was specific to MTB putative secretion system-associated gene clusters. This probe was a thiol-modified oligonucleotide at the $5^{\prime}$ end to form an $\mathrm{SH}$ group and gold atom with formation of an S-Au bond interaction. The thiol-labelling probe was synthesised from Bio Basic Canada Inc.

2.3. LAMP Amplification of the Target katG Gene in M. tuberculosis. A final volume of $25 \mu \mathrm{L}$ was used for the reactions, with contents comprising $0.2 \mathrm{mM}$ of each of the outer primers $\mathrm{F} 3$ and $\mathrm{B} 3,2 \mathrm{mM}$ of each of the inner primers FIP and BIP, and also 0.8-2.0 mM of dNTP mix (Promega, Madison, WI, USA), 1x of the supplied buffer (New England Biolabs Inc., Beverly, MA, USA), 0.6 M betaine (Sigma-Aldrich, St. Louis, MO, USA), 4-10 mM $\mathrm{MgSO}_{4}, 2.5-5.0 \mathrm{U}$ of Bst 2.0 warm start DNA polymerase (large fragment; New England Biolabs Inc., Beverly, MA, USA), and $1 \mu \mathrm{L}$ of template plasmid DNA (tenfold serial dilutions from $10^{-0}$ to $10^{-5}$ of total DNA of MDR-TB and standard strain H37Rv) in a final volume of $25 \mu \mathrm{L}$. A reaction mixture containing distilled water was used as negative control. The reaction mixture was incubated for $60 \mathrm{~min}$ at $61^{\circ} \mathrm{C}$, before subsequently undergoing $2 \mathrm{~min}$ of heating at $95^{\circ} \mathrm{C}$ in order to deactivate the enzyme and thus cause the reaction to stop. The resulting LAMP products were then stored at a temperature of $4^{\circ} \mathrm{C}$. They were analysed for LAMP products by $2 \%$ agarose gel electrophoresis in a $0.5 \mathrm{x}$ Tris Borate EDTA (TBE) buffer at 100 volts and observed under a Maestrogen Ultra Slim-LED blue light transilluminator by MaestroSafe Nucleic Acid Gel Stain Sample (Maestrogen).

2.4. Gold-Nanoparticle Synthesis. Prior to the preparation of AuNP preparation, the glassware involved underwent treatment using aqua regia (Sigma-Aldrich) in order to alleviate the problems caused when AuNPs stick to the vial surfaces, thereby lowering the effective concentrations in the vials. AuNPs were prepared by modifying a method used by Liu and Yi [11] and Suebsing et al. [12]. Briefly, $1 \mathrm{mM}$ hydrogen tretrachloroaurate trihydrate (Sigma-Aldrich) was stirred and mixed in $250 \mathrm{~mL}$ of distilled water while vigorously boiling prior to adding $38.8 \mathrm{mM}$ of sodium citrate tribasic dihydrate 


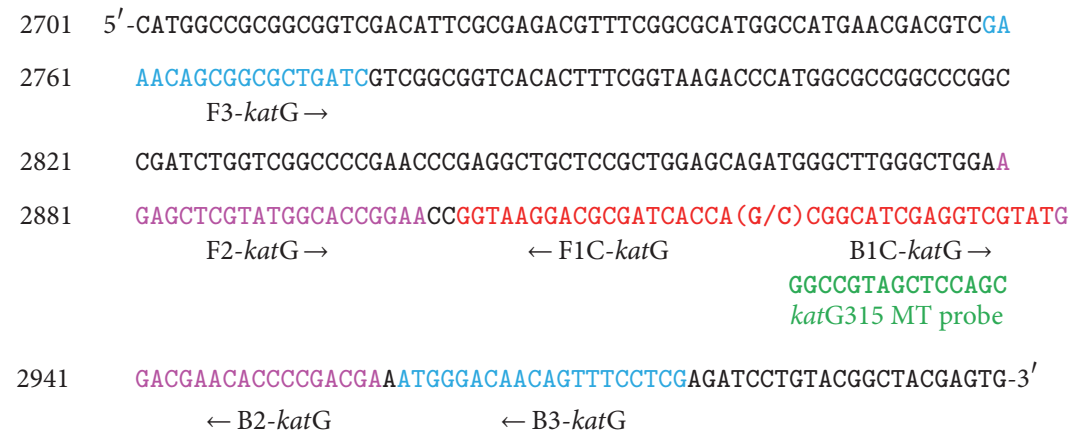

FIGURE 1: The directions and details of LAMP primers for amplification of a specific kat gene of M. tuberculosis.

TABLE 1: LAMP primers and Au-nanoprobe sequences.

\begin{tabular}{lrc}
\hline Primers/probe & ${\text { Nucleotide sequence } 5^{\prime} \text { to } 3^{\prime}}$ & Numbers of base $(\mathrm{bp})$ \\
\hline katG315 MT probe & $5^{\prime}$-Thiol-AAAAAAAAAACGACCTCGATGCCGG-3' & 15 \\
F3-katG & $5^{\prime}$-GAAACAGCGGCGCTGATC-3 & 18 \\
B3-katG & $5^{\prime}$-CGAGGAAACTGTTGTCCCAT-3' & 20 \\
FIP-katG & $5^{\prime}$-GTGGTGATCGCGTCCTTACCTTTTAGAGCTCGTATGGCACCGGAA-3' & 45 \\
BIP-katG & $5^{\prime}$-CTGGCATCGAGGTCGTATTTTTTCGTCGGGGTGTTCGTCC-3' & 40 \\
\hline
\end{tabular}

(Sigma-Aldrich). The solution was subsequently permitted to cool until reaching room temperature. The cooling phases were marked by a colour change in the solution, starting from yellow and then turning to clear, before changing again to black, then purple, and finally a deep red. The diameter of the AuNPs was about $15 \mathrm{~nm}$ with a surface plasmon band centre at $520 \mathrm{~nm}$. It was stored at $4^{\circ} \mathrm{C}$ prior to use.

All reagents and solvents were of the highest purity. Millipore MilliQ water $\left(18 \mathrm{M} \Omega \mathrm{cm}^{-1}\right)$ was used in all experiments.

2.5. Au-Nanoprobe Conjugated with a DNA Probe. The thiolated DNA probe was conjugated with Au-nanoparticles using a method reported by Mirkin et al. [13]. Initially, $20 \mu \mathrm{L}$ of the $100 \mu \mathrm{M}$ thiolated DNA probe was incubated with $4 \mathrm{~mL}$ of $10 \mathrm{nM}$ Au-nanoparticles in a shaker at $100 \mathrm{rpm}$ and $45^{\circ} \mathrm{C}$ for 24 hours in the dark. Then, the solution was added

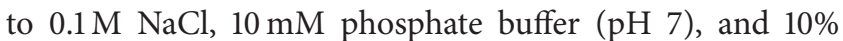
SDS. Subsequently, it was shaken at $100 \mathrm{rpm}$ at $45^{\circ} \mathrm{C}$ for 48 hours. It was centrifuged at $13,000 \mathrm{rpm}$ for $30 \mathrm{~min}$ to remove excess reagents. The precipitate was washed twice with $500 \mu \mathrm{L}$ of resuspension buffer containing $10 \mathrm{mM}$ PBS ( $\mathrm{pH} 7.4$ ), $150 \mathrm{mM} \mathrm{NaCl}$, and $0.1 \%$ SDS, after which it was resuspended in $50 \mu \mathrm{L}$ of the same buffer. The fully functionalised DNA$\mathrm{Au}$-nanoparticle conjugations ( $\mathrm{Au}$-nanoprobes) retained the same colour as the unmodified Au-nanoparticles with no visible aggregates. It was stored at $4^{\circ} \mathrm{C}$ until used.

The Au-nanoprobe solution was confirmed by UV/Vis spectroscopy. The peak for Au-nanoprobes was at $520 \mathrm{~nm}$ and its calculated concentration was the optimal amount for detection, which was approximately $10.8 \mathrm{nM}$.

2.6. Optimal Hybridisation of Au-Nanoprobes with LAMP Amplicons. For hybridisation, diluted LAMP products, from 1 to $9 \mu \mathrm{L}$ each, were mixed with the diluted Au-nanoprobes complex in equal volume. The mixture was hybridised at $61^{\circ} \mathrm{C}$ for $10 \mathrm{~min}$. After hybridisation, $0.7 \mathrm{M} \mathrm{MgSO}_{4}$ was added to the mixture in equal volume to induce aggregation of the $\mathrm{Au}$-nanoprobes at room temperature for $5 \mathrm{~min}$. The solution's colour change was discerned by direct observation. The complex solutions of Au-nanoprobe/LAMP products were determined by UV/vis spectra at $520 \mathrm{~nm}$ for confirmation.

2.7. Optimal Concentration of $\mathrm{MgSO}_{4}$ for Detection by Colorimetric Assay. The optimal concentration of $\mathrm{MgSO}_{4}$ for detection by colorimetric assay was determined. After hybridisation, $5 \mu \mathrm{L}$ of $\mathrm{MgSO}_{4}$ was added variously from $0.3,0.5$, and $0.7 \mathrm{M}$ to the mixture in equal volume to induce aggregation of $\mathrm{Au}-$ nanoprobes at room temperature for $5 \mathrm{~min}$. The solution's colour change was witnessed by direct observation.

2.8. Detection by Colorimetric Assay. Colorimetric assay was performed in a final volume of $15 \mu \mathrm{L}$ containing a fixed volume at $5 \mu \mathrm{L}$ of LAMP products from samples mixed with $5 \mu \mathrm{L}$ of Au-nanoprobe complex. The mixture was hybridised at $61^{\circ} \mathrm{C}$ for $10 \mathrm{~min}$. After hybridisation, $5 \mu \mathrm{L}$ of $0.7 \mathrm{M} \mathrm{MgSO}_{4}$ was added to the mixture to induce aggregation of Aunanoprobes at room temperature for $5 \mathrm{~min}$. The solution's colour change was witnessed by direct observation. The complex solutions of Au-nanoprobe/LAMP products were determined by UV/vis spectra at $520 \mathrm{~nm}$ for confirmation. The positive samples were observed as being of a reddishpurple colour, whereas the negative samples were observed as being of a bluish colour. Water was used as negative control. If the water was observed to be reddish-purple, the performance of the system was considered invalid.

2.9. Clinical Samples Test. The forty-six clinical isolates used were obtained from tuberculosis cluster, Bureau of AIDS-TBSTIs, including 31 samples of TB with resistance to INH and 15 susceptible to INH. Additionally, one strain of H37RV was used as negative control. Genotype MDR-TB Plus detection 
was confirmed after conventional methods detected cultures on a Lowenstein-Jensen (L.-J.) medium after 8 weeks. DNA was extracted from cultures with the modified method mentioned previously (Rienthong et al.) and used for LAMP amplification. The LAMP combined Au-nanoprobe assays used for identification of 46 clinical samples in this study were detected individually using the colorimetric assay compared to the standard Genotype MDR-TB Plus assays.

\section{Results}

The authors created a two-stage approach with its foundation in both the molecular signatures of the MDR-TB members and also the most widely encountered mutations related to INH resistance in MTB. The key step of the process involved the LAMP amplification of a kat $\mathrm{G}$ gene fragment followed by its hybridisation using specific Au-nanoprobes. The kat $\mathrm{G}$ locus shared by all members of MDR-TB were targeted and a probe specific to MDR-TB members was designed (katG315 MT probe). One additional set of probes specific to the most common point mutations associated with INH resistance (katG315) was also synthesised. Each set was composed of the complement to the mutation.

3.1. Optimal LAMP INHr-TB Reaction. The researchers determined the optimal temperature for LAMP INHr-TB reaction to detect a specific kat $\mathrm{G}$ gene of $\mathrm{MDR}-\mathrm{TB}$. The optimisation of LAMP products detected in the optimum temperature was carried out at $61^{\circ} \mathrm{C}$ (Figure 2) and the optimal concentration of $\mathrm{MgSO}_{4}$ was used for the LAMP reaction to detect the specific kat $\mathrm{G}$ gene of MDR-TB. The LAMP reaction was carried out at $4 \mathrm{mM} \mathrm{MgSO}_{4}$. The optimum concentration of dNTP for LAMP reaction was carried out at $1.6 \mathrm{mM}$ of concentration dNTP. The LAMP detection of MDR-TB showed a limited condition at $10^{-3}$ dilution of the DNA templates (genomic DNA) for LAMP tests performed under optimised conditions (Figure 3).

3.2. Optimal Concentration of $\mathrm{MgSO}_{4}$ for Detection by Colorimetric Assay. $\mathrm{MgSO}_{4}$ concentration was used for detection by colorimetric assay of a specific katG gene of MDR-TB. The positive samples of a reddish-purple colour (no colour

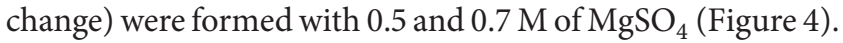
For colorimetric assay, the colorimetric reaction was carried out with $0.7 \mathrm{M}$ of $\mathrm{MgSO}_{4}$ concentration and the colorimetric reaction was followed with the concentration described above. The solution's colour change was witnessed by direct observation.

3.3. Optimal Hybridisation for Au-Nanoprobe Assay. The researchers determined the optimal hybridisation of $5 \mu \mathrm{L}$ LAMP products with $5 \mu \mathrm{L}$ of Au-nanoprobe complex. The mixture was hybridised at $61^{\circ} \mathrm{C}$ for $10 \mathrm{~min}$. Then, $5 \mu \mathrm{L}$ of $0.7 \mathrm{M}$ $\mathrm{MgSO}_{4}$ was added to induce aggregation of Au-nanoprobes at room temperature for $5 \mathrm{~min}$. The solution's colour change was observed directly (Figure 5). These conditions were used for the assay.

3.4. Au-Nanoprobes Colorimetric Assay. Solutions containing the Au-nanoprobe revealed a red colouration due to

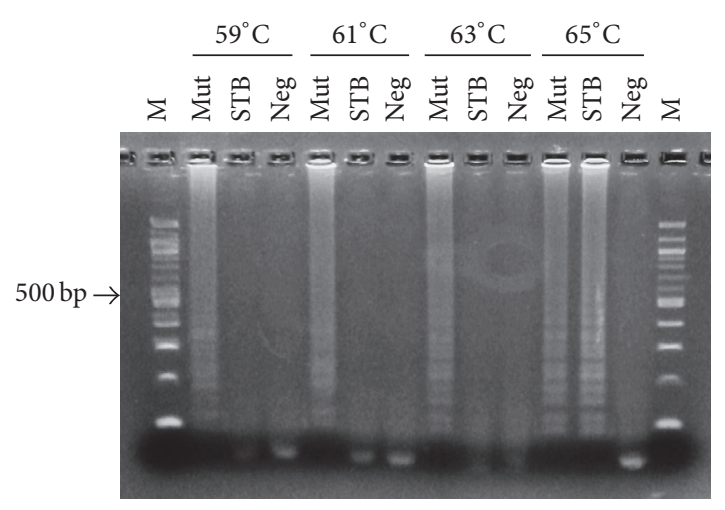

FIGURE 2: Determining the optimal temperature for LAMP. Lanes 1 and $14(\mathrm{M})$ : Ladder DNA marker; Lanes 2-13: reaction for $60 \mathrm{~min}$ at $59,61,63$, and $65^{\circ} \mathrm{C}$; Mut as MDR-TB; STB as H37RV; Neg as negative. All LAMP products were electrophoresed on $2 \%$ agarose gels and stained with MaestroSafe Nucleic Acid Gel Stain Sample (Maestrogen).

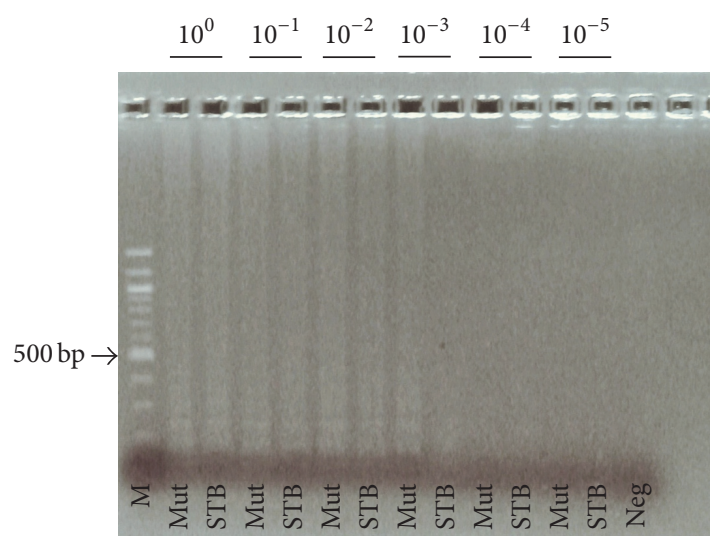

(a)

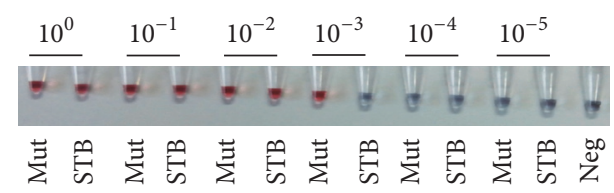

(b)

FIgURE 3: Determining the DNA templates for LAMP of MDRTB members via the Au-nanoprobes assay. (a) Agarose gel showed LAMP products. Lane $1(\mathrm{M})$ : Ladder DNA marker; Lanes 1-12: DNA templates for LAMP at $10^{0}-10^{-5}$, Mut as MDR-TB, STB as H37RV, and Neg as negative. All LAMP products were electrophoresed on $2 \%$ agarose gels and stained with MaestroSafe Nucleic Acid Gel Stain Sample (Maestrogen). (b) Corresponding results recorded visually after salt challenge. Red colour showed the presence of the target and purple indicated its absence.

the localised surface plasmon resonance (SPR) of the Aunanoprobes. When the complementary target was present, these Au-nanoprobes had protection from aggregation induced by salt, and therefore the solution did not change colour. However, if a noncomplementary target was present, no protection existed and the solution duly changed to 


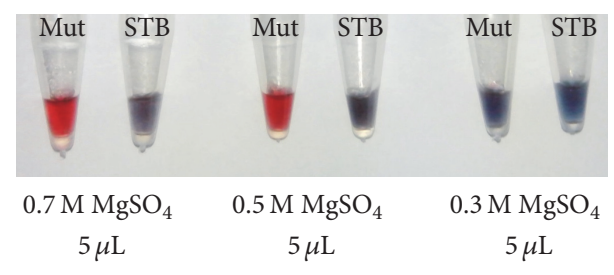

FIGURE 4: Determining the optimal concentration of $\mathrm{MgSO}_{4}$ for detection by colorimetric assay.

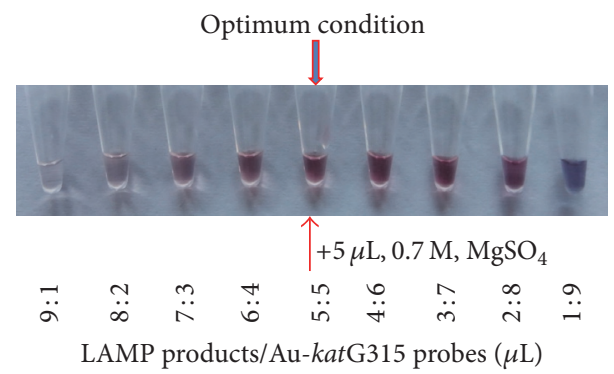

FIGURE 5: Determining the optimal condition of hybridisation for Au-nanoprobe assay.

blue (Figures 6(a)-6(b)). The data derived from UV-Vis spectroscopy performed on the samples as shown in Figure 6(c) support the hypothesis related to visual discrimination of the sample being aggregation induced. The sample which contained MDR-TB genomic DNA clearly revealed an absorbance peak characteristic of Au-nanoprobes at $520 \mathrm{~nm}$. This was because the free conduction band electrons of the dispersed particles become collectively excited. In the control samples and negative samples, the existence of a broad absorbance spectrum indicated a peak shift denoting a longer wavelength $(\geq 600 \mathrm{~nm})$ as the particles in the aggregates undergo coupling.

3.5. Application of the LAMP Combined Au-Nanoprobes Assay for INHr-TB Detection. All clinical samples were used for detection of the kat $\mathrm{G}$ gene (31 positive samples and 15 negative samples were confirmed by Genotype MDR-TB Plus), which were then assayed for positive sample presence of polymorphism in codons 315 of the $k a t \mathrm{G}$ gene $(\mathrm{g} \rightarrow \mathrm{c})$ (Figures 7-10). The Au-nanoprobe results were compared to those obtained via Genotype MDR-TB Plus summary in Table 2. The Au-nanoprobe assay showed sensitivity and specificity at $100 \%$ in a test compared with Genotype MDR-TB Plus.

\section{Discussion}

On the basis of the MDT-TB members' molecular signatures along with the most frequently observed mutations linked with INH resistance in $M$. tuberculosis, the authors established a two-stage process involving LAMP amplification of a $k a t \mathrm{G}$ gene fragment followed by hybridisation using specific Au-nanoprobes. The kat $\mathrm{G}$ locus shared by all members of MDR-TB was targeted and a probe specific to INHr-TB members was designed ( $k a t \mathrm{G} 315$ probe).

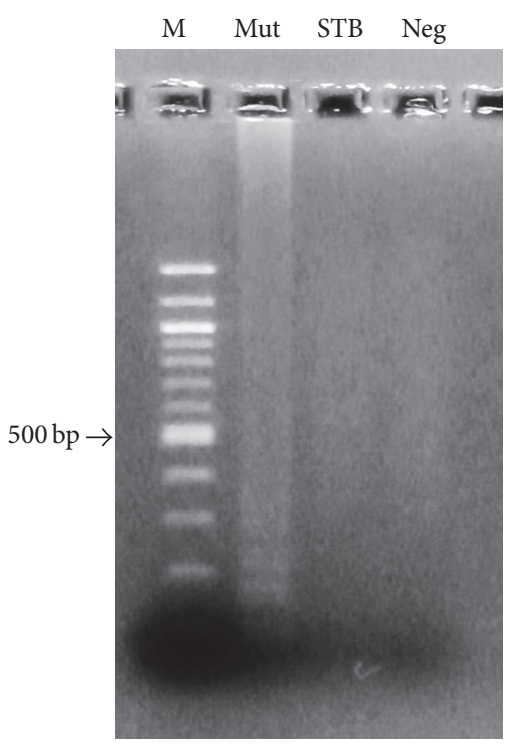

(a)

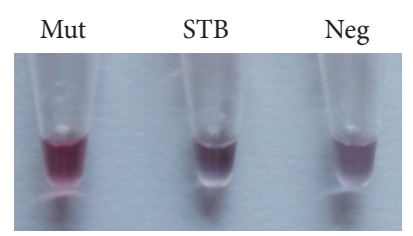

(b)

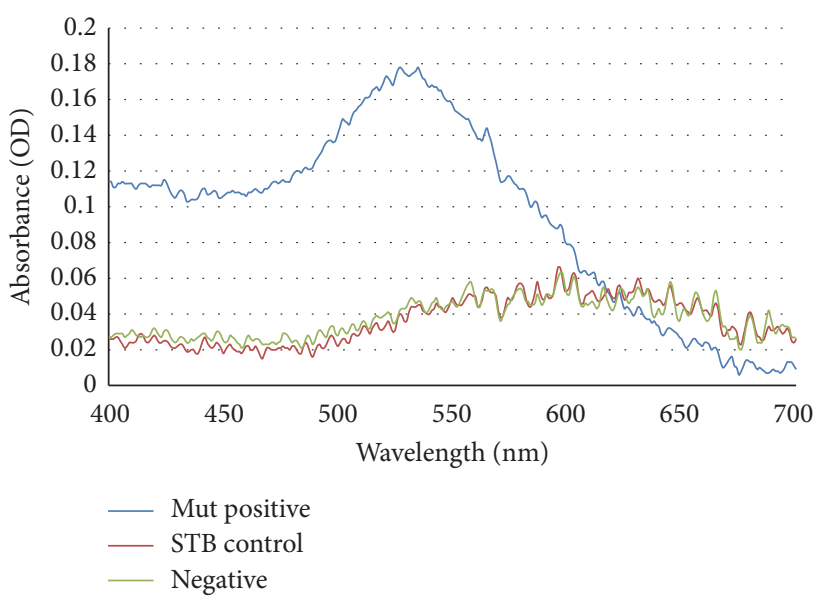

(c)

FIgURE 6: Au-nanoprobe colorimetric assay. (a) Agarose gel showing LAMP products. Lane 1, M: Ladder DNA marker; Lane 2: Mut (MDR-TB), Lane 3: STB (Standard strain H37RV), and Lane 4: Neg (negative). (b) Corresponding results recorded visually after salt challenge. (c) UV-Vis spectra of the respective samples.

In this study, the researchers designed a strategy based on the LAMP of DNA samples coupled to specific Aunanoprobes. It showed the potential to provide a rapid and specific method for detection of isoniazid resistance (INHr) at $k a t \mathrm{G}$ gene position $315(\mathrm{G} \rightarrow \mathrm{C})$. The researchers determined the optimal temperature for LAMP reaction in the detection of specific kat $\mathrm{G}$ gene of $\mathrm{INHr}-\mathrm{TB}$ at $61^{\circ} \mathrm{C}$ for 


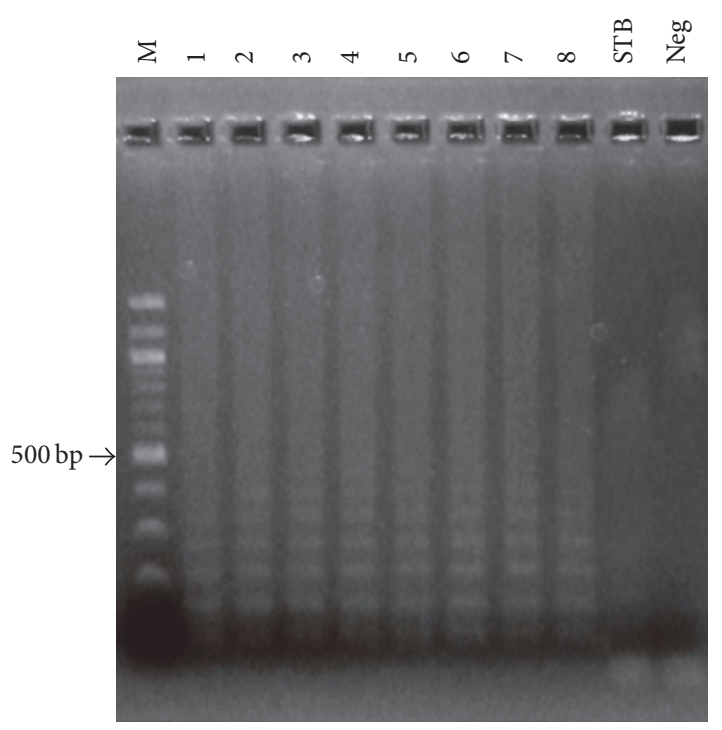

(a)

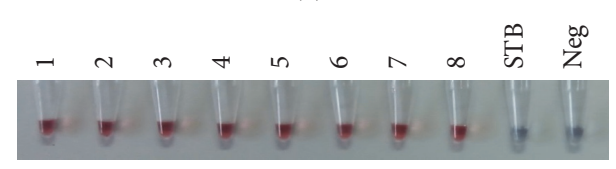

(b)

FIGURE 7: Au-nanoprobe colorimetric assay for MDR-TB detection. (a) Agarose gel showed LAMP products. Lane M: Ladder DNA marker; Lanes 1-8: Mut (MDR-TB), Lane STB: standard strain H37RV, and Lane Neg: negative. (b) Corresponding results recorded visually after salt challenge. A red colour showed the presence of the target, while purple indicated its absence.

TABLE 2: MDR-TB member detection using the Au-nanoprobe assay. Test results from the Au-nanoprobes assay were subsequently compared with results from the Genotype MDR-TB Plus assay.

\begin{tabular}{lccc}
\hline \multirow{2}{*}{$\begin{array}{l}\text { Genotype }{ }^{\circledR} \text { MDRTB Plus } \\
\text { TB assay }\end{array}$} & \multicolumn{3}{c}{ Au-nanoprobes assay kat G315 probe } \\
& Positive & Negative & Total \\
\hline INH (315) resistant (31) & 31 & 0 & 31 \\
INH susceptible (15) & 0 & 15 & 15 \\
Total (46) & 31 & 15 & 46 \\
\hline
\end{tabular}

$60 \mathrm{~min}$. The $\mathrm{MgSO}_{4}$ and dNTP concentrations were optimised because they were able to influence LAMP reactions, as described previously [2]. It was also found that at least $4 \mathrm{mM} \mathrm{MgSO}_{4}$ was required in the LAMP reaction, although inhibition could result in higher than $10 \mathrm{mM} \mathrm{MgSO}_{4}$ due to reduced activity of the Bst 2.0 warm start DNA polymerase and destabilisation of the DNA helix [2]. The dNTP mix at $0.8 \mathrm{mM}$ was minimal for the LAMP reaction under the optimal $\mathrm{MgSO}_{4}$ concentration. The researchers found the optimal condition for LAMP reaction at 4-6 $\mathrm{mM} \mathrm{MgSO}_{4}$, 0.8-1.6 mM dNTP mix, and 2.5-5.0 U of Bst 2.0 warm start DNA polymerase to be suitable for detection of drug resistance, which is a one-point mutation associated with INHr in MTB. The determination of DNA templates for LAMP of MDR-TB members was via the Au-nanoprobe assay. The

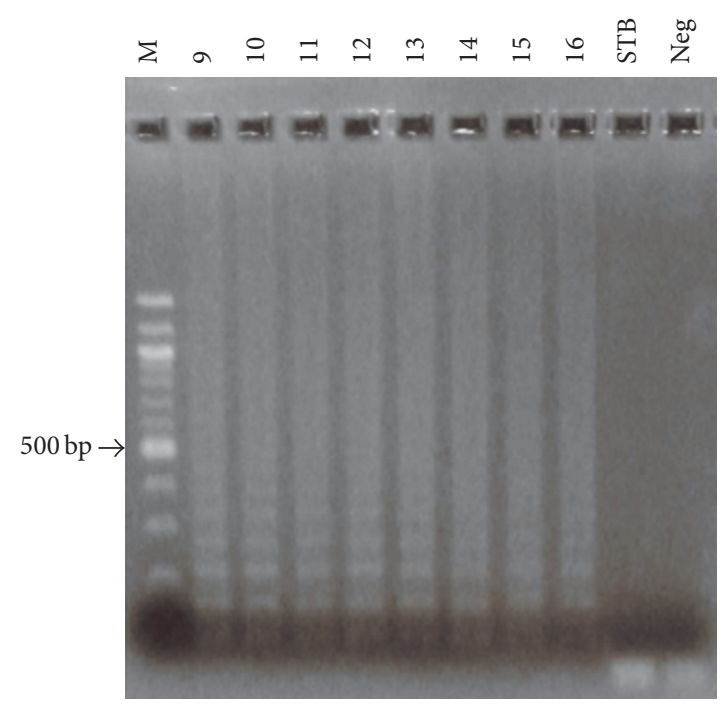

(a)

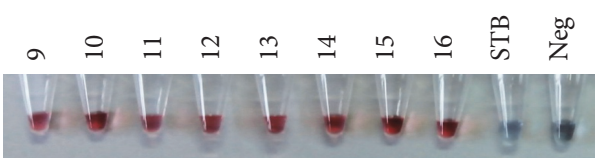

(b)

FIGURE 8: Au-nanoprobe colorimetric assay for MDR-TB detection. (a) Agarose gel showed LAMP products. Lane M: Ladder DNA marker; Lanes 9-16: Mut (MDR-TB), Lane STB: standard strain H37RV, and Lane Neg: negative. (b) Corresponding results recorded visually after salt challenge. Red colour showed the presence of the target, while purple indicated its absence.

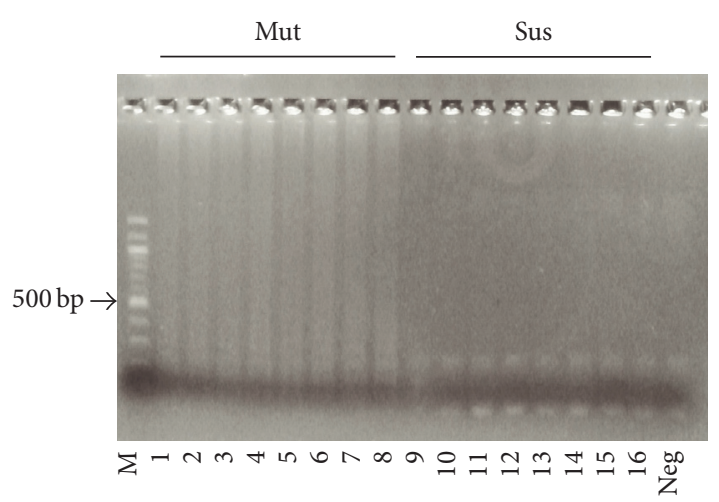

(a)

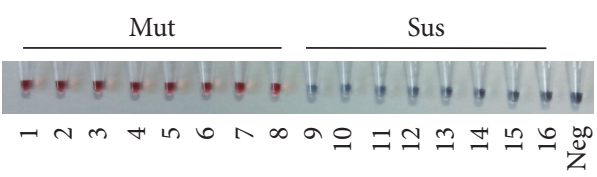

(b)

FIGURE 9: Detection of MDR-TB members via Au-nanoprobe assay. (a) Agarose gel showed LAMP products. Lane $1(\mathrm{M})$ : Ladder DNA marker; Lanes 1-16: Mut as MDR-TB, Sus as INH susceptible, and Neg as negative. (b) Corresponding results recorded visually after salt challenge. Red colour showed the presence of the target, while purple indicated its absence. 


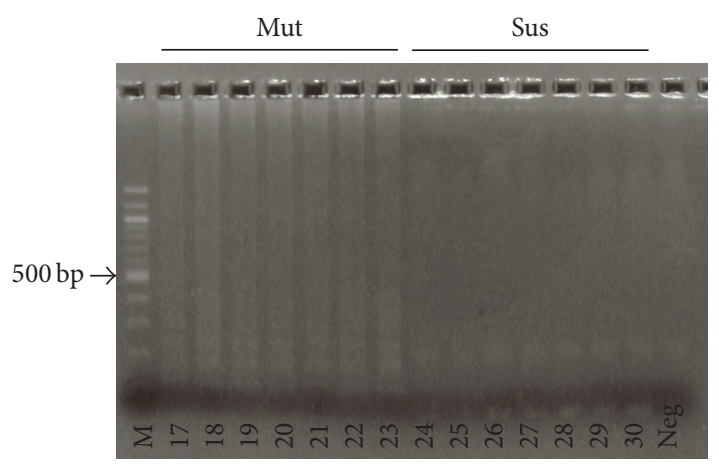

(a)

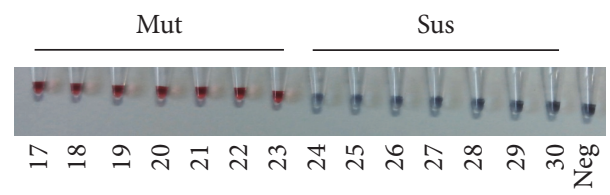

(b)

FIGURE 10: Detection of MDR-TB members via Au-nanoprobe assay. (a) Agarose gel showed LAMP products. Lane $1(\mathrm{M})$ : Ladder DNA marker; Lanes 17-30: Mut as MDR-TB, Sus as INH susceptible, and Neg as negative. (b) Corresponding results recorded visually after salt challenge. Red colour showed the presence of the target, while purple indicated its absence.

LAMP detection of MDR-TB showed a limited condition at $10^{-3}$ dilution of the DNA templates (genomic DNA) for LAMP tests performed under optimised conditions. This corresponded to the detection of DNA template limits for LAMP of MDR-TB members via the Au-nanoprobe assay.

Specific hybridisation between the LAMP amplicons combined with Au-nanoprobes resulted in the formation of a three-dimensional polymeric network of non-cross-linked Au-nanoprobes that prevented them from forming close aggregates. Thus, they maintained their deep red colouration when salt was present. However, when the Au-nanoprobes were left unprotected, aggregation took place when salt was present in the blank and in the negative control which contained the noncomplementary DNA. The absorbance spectrum consequently experienced a quantum shift, which could be observed in the form of a colour change to blue from the original red [8]. However, this method was 2-step LAMP test; we must open the cover of the reaction tube in order to add Au-nanoprobes. In this time, there is the risk of the contamination of the LAMP products in other samples. For this reason, the Au-nanoprobe reacted with LAMP amplicons contamination could not overcome negative result that contamination is a rare event. Moreover, the LAMP amplification inactivated the enzyme by heating at $95^{\circ} \mathrm{C}$ for $2 \mathrm{~min}$ in terminating the reaction. The optimisation tests revealed the importance of the ratio between LAMP amplicons and Au-nanoprobes, since this would influence whether the detection of hybridisation between Au-nanoprobes and LAMP amplicons would succeed and also whether it might be reproducible. The most suitable ratio was shown to be $5: 5 \mu \mathrm{L}$. The salt concentration $\left(5 \mu \mathrm{L}\right.$ of $0.7 \mathrm{mM} \mathrm{MgSO}_{4}$ ) used to promote aggregation after the hybridisation step was critical as well. In addition to colorimetric reaction, confirmation of the LAMP products/Au-nanoprobe complex solutions was determined by UV/vis spectra at $520 \mathrm{~nm}$ for confirmation.

The Au-nanoprobe system proposed in this study was capable of detecting MDR-TB members and mutations associated with isoniazid resistance, while also being easy to perform without the need for expensive and complex laboratory set-ups previously studied [14]. It is important that the MDR-TB members and mutations within the kat $\mathrm{G}$ gene should be quickly and accurately identified, since this aids the predication, at a high confidence level, of whether or not the strain under examination is MDR-TB.

In summary, the researchers designed a strategy based on Au-nanoprobe detection protocol combined with LAMP for the rapid detection of MDR-TB strains as well as simultaneous characterisation of the presence of mutations associated with isoniazid resistance. In field analysis, it was shown that the application of LAMP to carry out the DNA sample amplification was highly appropriate since the greater specificity and speed, along with the isothermal profile, resulted in a more effective approach in comparison with standard PCR. This method was advantageous because it was quick, cheap, specific, and sensitive for MDR-TB detection. It could be convenient for the detection of MDR-TB in field samples as well.

\section{Conclusions}

The goal of creating simple but robust portable platforms for molecular diagnosis has attracted considerable attention, since it will support the fight against TB in peripheral laboratories and at point-of-care facilities. The Au-nanoprobe system proposed in this research was capable of detecting not only MDR-TB members but also mutations which are linked with isoniazid resistance (INHr). It offered the advantage of being simple to use and did not require expensive wellequipped laboratory facilities. MDR-TB members and mutations within the kat $G$ gene could be quickly and accurately identified, which represents a significant advantage since it allows predictions regarding the identity of a strain to be made with a higher degree of confidence. This ensures that patients carrying MDR-TB can be quickly diagnosed, isolated, and treated, thereby reducing the problem of noncompliance. In tests, it was shown that, in comparison with the Genotype MDR-TB Plus TB assay, Au-nanoprobes were able to correctly confirm the presence MDR-TB DNA in 100\% of samples. Following LAMP amplification, the combined $\mathrm{Au}$-nanoprobe system took less than $75 \mathrm{~min}$ to yield a colorimetric result using a suitable eye detector. In addition, tests were carried out to assess the potential use through a portable assay platform, confirming the suitability of the approach for field screening. Further studies may be required in order to optimise the validation methodology and to determine the best approach for the direct application to clinical samples. The concept should also be extended to be applied in the case of other mutations which have been linked to drug resistance. 


\section{Competing Interests}

The authors declare that they have no competing interests.

\section{Acknowledgments}

This work was supported by Srinakharinwirot University. Additionally, the researchers would like to acknowledge the assistance provided by the Bureau of Tuberculosis in the Thai Ministry of Public Health.

\section{References}

[1] World Health Organisation, "Global tuberculosis control," 2012, http://www.who.int/tb/publications/global_report/en/index .html.

[2] T. Notomi, H. Okayama, H. Masubuchi et al., "Loop-mediated isothermal amplification of DNA," Nucleic Acids Research, vol. 28, no. 12, article E63, 2000.

[3] S. Ikeda, K. Takabe, M. Inagaki, N. Funakoshi, and K. Suzuki, "Detection of gene point mutation in paraffin sections using in situ loop-mediated isothermal amplification," Pathology International, vol. 57, no. 9, pp. 594-599, 2007.

[4] Y. Mori, K. Nagamine, N. Tomita, and T. Notomi, "Detection of loop-mediated isothermal amplification reaction by turbidity derived from magnesium pyrophosphate formation," Biochemical and Biophysical Research Communications, vol. 289, no. 1, pp. 150-154, 2001.

[5] M. Iwasaki, T. Yonekawa, K. Otsuka et al., "Validation of the loop-mediated isothermal amplification method for single nucleotide polymorphism genotyping with whole blood," Genome Letters, vol. 2, no. 3, pp. 119-126, 2003.

[6] A. Badolo, K. Okado, W. M. Guelbeogo et al., "Development of an allele-specific, loop-mediated, isothermal amplification method (AS-LAMP) to detect the L1014F $k d r$ - $w$ mutation in Anopheles gambiae s. l.," Malaria Journal, vol. 11, article 227, 2012.

[7] P. V. Baptista, E. Pereira, P. Eaton et al., "Gold nanoparticles for the development of clinical diagnosis methods," Analytical and Bioanalytical Chemistry, vol. 391, no. 3, pp. 943-950, 2008.

[8] T. Kaewphinit, S. Santiwatanakul, and K. Chansiri, "Colorimetric DNA based biosensor combined with loop-mediated isothermal amplification for detection of Mycobacterium tuberculosis by using gold nanoprobe aggregation," Sensors \& Transducers, vol. 142, pp. 123-128, 2013.

[9] B. Veigas, P. Pedrosa, I. Couto, M. Viveiros, and P. V. Baptista, "Isothermal DNA amplification coupled to Au-nanoprobes for detection of mutations associated to Rifampicin resistance in Mycobacterium tuberculosis," Journal of Nanobiotechnology, vol. 11, no. 1, article 38, 2013.

[10] D. Rienthong, S. Rienthong, C. Boonin, S. Woraswad, and Y. Kasetjaroen, "Rapid detection for early appearance of rifampin and isoniazid resistance in Mycobacterium tuberculosis," Siriraj Medical Journal, vol. 61, pp. 49-55, 2009.

[11] J. Liu and L. Yi, "Preparation of aptamer-linked gold nanoparticle purple aggregates for colorimetric sensing of analytes," Nature Protocols, vol. 1, no. 1, pp. 246-252, 2006.

[12] R. Suebsing, P. Prombun, J. Srisala, and W. Kiatpathomchai, "Loop-mediated isothermal amplification combined with colorimetric nanogold for detection of the microsporidian Enterocytozoon hepatopenaei in penaeid shrimp," Journal of Applied Microbiology, vol. 114, no. 5, pp. 1254-1263, 2013.
[13] C. A. Mirkin, R. L. Letsinger, R. C. Mucic, and J. J. Storhoff, "A DNA-based method for rationally assembling nanoparticles into macroscopic materials," Nature, vol. 382, no. 6592, pp. 607609, 1996.

[14] B. Veigas, D. Machado, J. Perdigão et al., "Au-nanoprobes for detection of SNPs associated with antibiotic resistance in Mycobacterium tuberculosis," Nanotechnology, vol. 21, no. 41, Article ID 415101, 2010. 

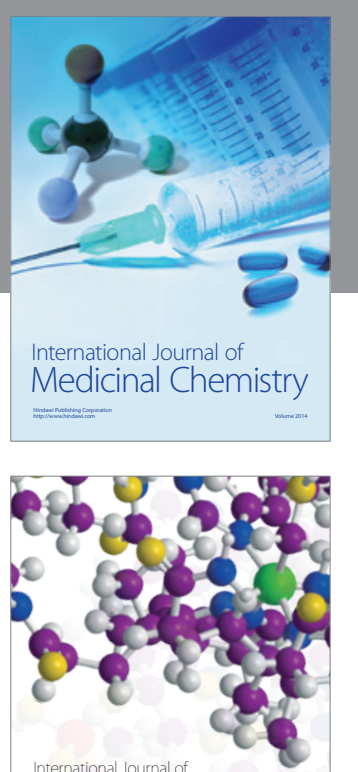

Carbohydrate Chemistry

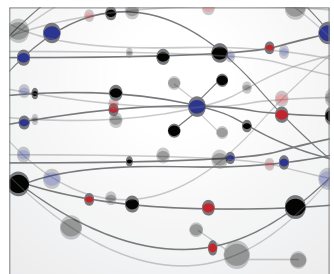

The Scientific World Journal
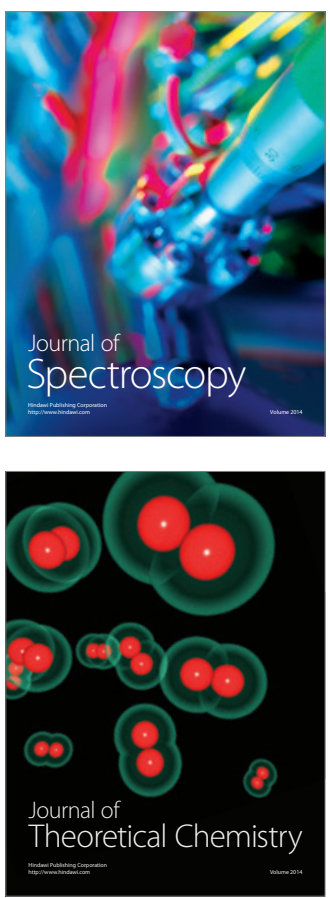
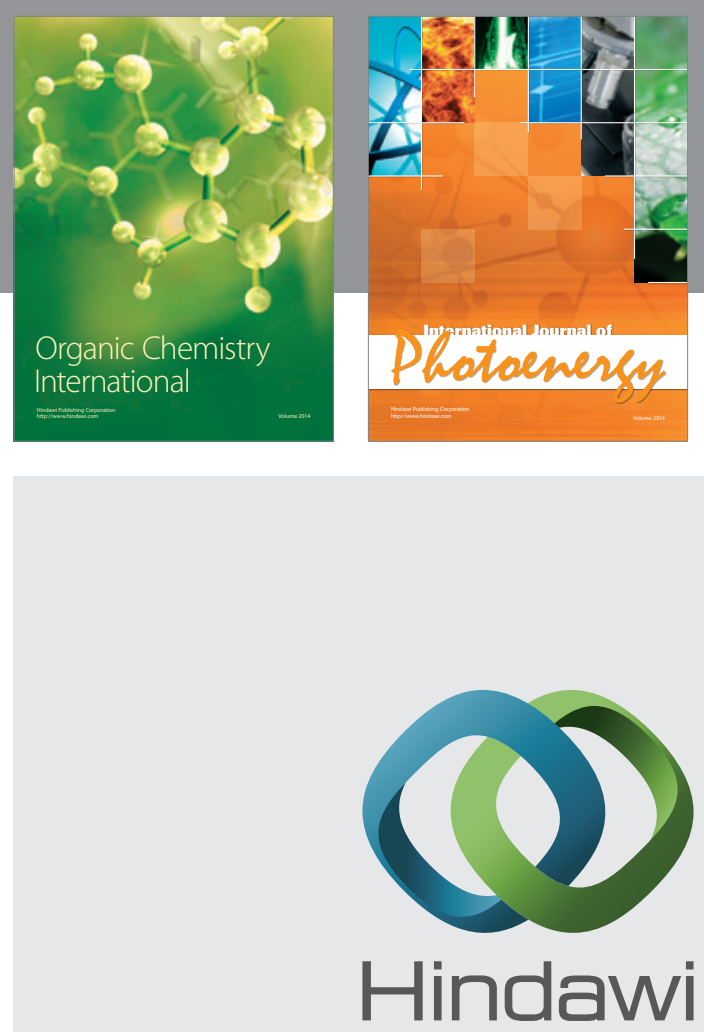

Submit your manuscripts at

http://www.hindawi.com

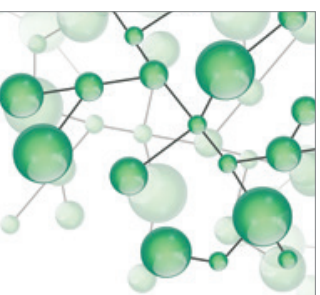

International Journal of

Inorganic Chemistry

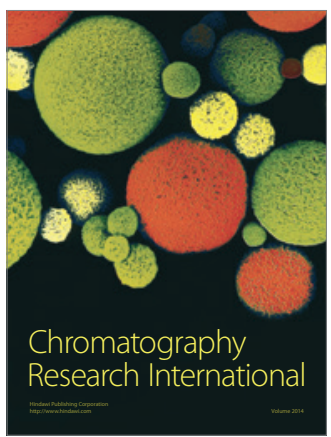

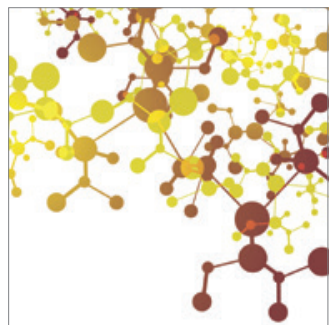

Applied Chemistry
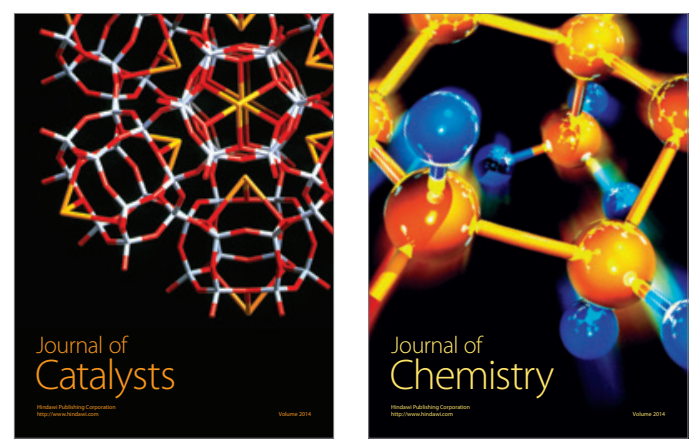
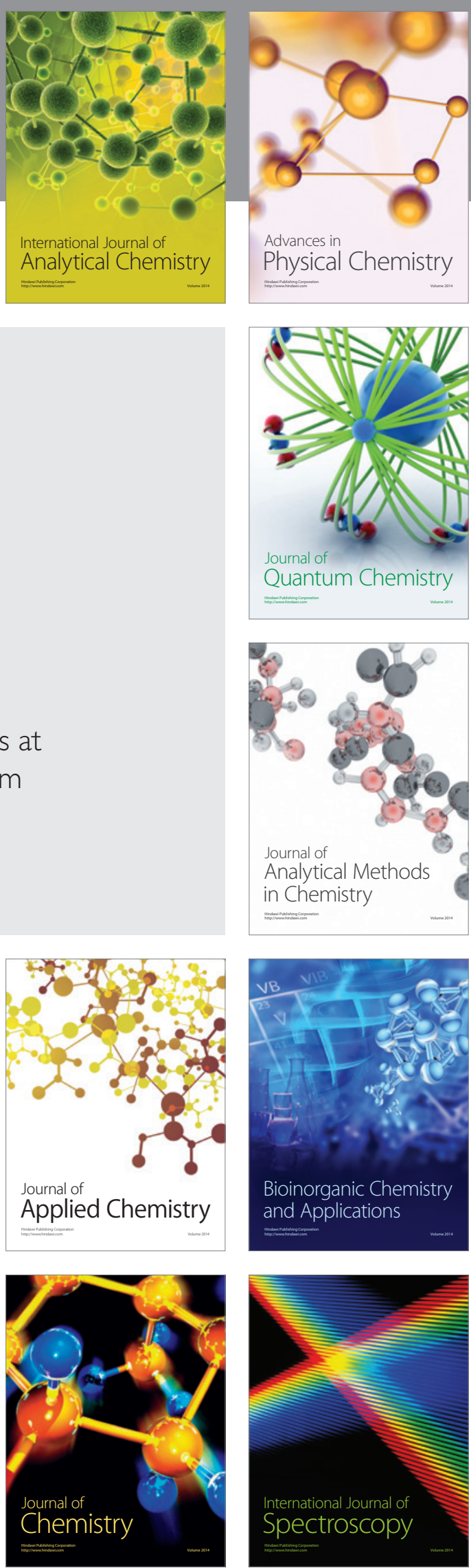